\title{
COMPETENCIAS TIC EN DOCENTES DEL INGLÉS A NIVEL DE BÁSICA PRIMARIA EN LAS IE OFICIALES DE LA REGIÓN CARIBE DE COLOMBIA
}

\author{
GIOVANNI POLIFRONI LOBO 1 \\ Universidad del Norte. Km. 5 Antigua Vía Puerto Colombia. \\ Barranquilla, Colombia \\ gpolifroni@mail.uniatlantico.edu.co
}

\section{RESUMEN}

El presente trabajo está referido de manera particular, a identificar la percepción de los docentes en relación con el uso de las TIC en las labores de enseñanza del inglés, y de forma general, a la profundización y el conocimiento en las prácticas pedagógicas en las instituciones de educación a nivel de básica primaria en el Departamento del Atlántico. El estudio es de carácter descriptivo. Se hizo una recolección de datos sobre las competencias de los docentes bilingües de la básica primaria en las escuelas públicas. Se aplicaron encuestas y se utilizó el software estadístico SPSS para el análisis de los datos. Se concluyó, que los docentes no están generando un nuevo conocimiento, debido a que no participan en la construcción de proyectos investigación apoyados por las nuevas tecnologías.

Palabras clave: Docentes, TIC, escuelas, bilingüe, básica primaria, Atlántico, Tecnología, estudiantes, profundización, generación, conocimiento.

\footnotetext{
${ }^{1}$ Magister en Comunicación Social. Docente Catedrático. Programa de Comunicación Social. Universidad del Norte.
} 
COMPETITIONS TIC ORIENTATED TO THE DEEPENING AND GENERATION OF THE KNOWLEDGE IN TEACHERS, AT THE EXPENSE OF THE EDUCATION OF THE ENGLISHMAN TO LEVEL OF BASIC PRIMARY IN THE OFFICIAL IE OF THE CARIBBEAN REGION OF COLOMBIA

ABSTRACT-

In particular, this work refers to identifying the perception of the teachers regarding the use of TICs in English teaching. In general, it discusses deepening and knowledge in the pedagogical practices of the institutions of education at the primary level in Departamento del Atlántico.

The study design is descriptive. It was performed a data collection related to de competences of the bilingual teachers in the primary level of the public schools. Surveys were applied and it was used the SPSS statistical software to analyze data. It was found that teachers were not generating new knowledge because they do not participate in the building of research projects supported by new technologies.

Key words: Teachers, ICT, schools, bilingual elementary school, Atlantic Technology students, deepening, generation, knowledge

\section{Introducción}

En la actualidad, las TIC están presentes en todos los órdenes de la vida en sociedad, por lo tanto, la educación no escapa a la influencia que este fenómeno. La introducción de estas nuevas tecnologías en la educación ha traído consigo una variedad de posibilidades en materia educativa, ante las oportunidades potenciales que brinda, tanto a docentes como estudiantes, la capacidad de adquisición de: habilidades de búsqueda de información, la simulación de fenómenos sin riesgo para solucionar problemas, el aprendizaje significativo, entre otros aspectos. El empleo de las TIC en el aula de clase, supone la convergencia de lo pedagógico, lo comunicativo y lo tecnológico, es decir, se trata del empleo correcto de las herramientas tecnológicas, posibilitando con ello, una adecuada interacción en el proceso educativo capaz de integrar lo discursivo y lo tecnológico. 
Según Robinson (2011), es posible personalizar la educación y atender a los intereses, habilidades y estilos de aprendizaje de todas las personas a través del uso creativo de nuevas tecnologías. El reto no es el proponer un solo modelo formativo a nivel general, sino propagar los principios de la creatividad en toda la educación, para que cada institución educativa desarrolle sus propias formas de afrontar los desafíos que enfrenta como comunidad singular.

La UNESCO, elaboró un marco de referencia para el desarrollo profesional docente con estándares de competencias para ayudar a los países a dar un enfoque integral a las TIC en la educación de manera que la formación de los docentes responda a la visión de país. Para lograr esta articulación aborda las TIC desde seis componentes (política y visión, currículo y evaluación, pedagogía, TIC, organización, y aprendizaje profesional del docente) y en tres etapas progresivas (alfabetización tecnológica, profundización del conocimiento y creación del conocimiento) (UNESCO, 2011).

Las competencias en TIC facilitan el camino a la generación de nuevos conocimientos. Para poder desarrollarlas el docente requiere además de una actitud positiva frente al tema, ser capacitado y contar con una infraestructura que le ofrezca y permita acceder a los recursos TIC. De ahí que, las universidades, escuelas o centros de formación posean parte de responsabilidad en esta labor (Mejía y Puche, 2008).

En relación a la profundización del conocimiento mediante las TIC, diferentes investigaciones han concluido que los docentes, casi nunca ponen en práctica mecanismos tecnológicos en su gestión de aula. Los valores se ubican en la categoría de competencias informáticas poco consolidadas: no promueven la interacción en aula a través de medios tecnológicos, no practican en el aula estrategias educativas en base al conocimiento digital, no manejan vocabulario tecnológico, no generan entornos de aprendizaje tecnológico que le permitan desarrollar habilidades y competencias suficientes en el área (Mendoza, citado por Mejía y Puche, 2009). El docente debe ser el encargado de construir ambientes innovadores. Debe seleccionar las estrategias y las TIC adecuadas 
para que entre los estudiantes se establezcan relaciones cooperativas, y se logre construir conocimiento aprendiendo unos de otros (MEN, 2013).

Una realidad evidente en el contexto de la región Caribe, es la falta de conocimiento que presentan los docentes de las instituciones de educación a nivel de básica primaria, en cuanto al uso de las tecnologías, y la importancia que tienen ellas, en cuanto al apoyo de las enseñanzas de los contenidos pedagógicos. Ante esta situación, se plantea como objetivo central, el analizar las competencias TIC orientadas a la profundización y generación del conocimiento en docentes, a cargo de la enseñanza del inglés a nivel de básica primaria en las instituciones de educación (IE) oficiales de la región Caribe. El presente trabajo está referido de manera particular, a identificar la percepción de los docentes en relación con el uso de las TIC en las labores de enseñanza del inglés, y de forma general, a la profundización y el conocimiento en las prácticas pedagógicas en las instituciones de educación a nivel de básica primaria en las instituciones de educación (IE) oficiales en el Departamento del Atlántico.

El presente trabajo está referido de manera particular, a identificar la percepción de los docentes en relación con el uso de las TIC en las labores de enseñanza del inglés, y de forma general, a la profundización y el conocimiento en las prácticas pedagógicas en las instituciones de educación a nivel de básica primaria en el Departamento del Atlántico.

\section{Preguntas de investigación}

¿Cuál es el perfil de uso de las TIC de los docentes a cargo de la enseñanza del inglés a nivel de Básica Primaria en las IE oficiales de la región Caribe de Colombia?

¿Qué tipo de percepción tienen los docentes a cargo de la enseñanza del inglés a nivel de Básica Primaria en las IE oficiales de la región Caribe de Colombia, en torno a la utilidad e implementación de las TIC en la labor de profundización y generación de conocimiento en los contenidos y contexto requeridos para el desarrollo de sus prácticas pedagógicas? 
¿Cuáles con los rasgos del uso de las TIC en las prácticas pedagógicas, ejercidas por los profesores estudiados?

\section{Planteamiento del Problema}

\subsection{Descripción del Problema}

Uno de los actores clave en el proceso de formación de los estudiantes al interior de las instituciones educativas, son los docentes. En cuanto a la realidad que tiene este colectivo a nivel nacional y regional (Caribe), se puede destacar la especial atención que debe hacerse en torno a la generación de mecanismos y acciones que ayuden al fortalecimiento de los métodos y competencias requeridos para la enseñanza ejercida por éstos.

En los últimos cinco años, el sector educativo de la región Caribe colombiana ha experimentado un proceso de aumento y consolidación de las posibilidades de acceso a las TIC. El número de equipos de cómputo en instituciones educativas oficiales de la región pasó de 23.388 en 2005 a 93.867 en 2010. En lo referente a la posibilidad de acceder a internet, en el 2010, 83 de cada 100 instituciones educativas oficiales de la región contaban con conexión a la internet. Asimismo, se destaca que el porcentaje de escuelas oficiales conectadas a internet en la región Caribe (83\%) es mayor que el reportado para el consolidado nacional (73\%) y el avance en conectividad de dichas instituciones entre 2005 y 2010 (52 puntos porcentuales) fue mayor que el consolidado Nacional (29 puntos porcentuales) (Hung, 2014). Sin embargo, a pesar de ello, se requiere la integración de las TIC en el currículo, ya que no se logra evidenciar información disponible acerca de la inclusión efectiva de las TIC en los currículos de las escuelas por parte de los profesores. Ello dificulta el diseño de acciones orientadas al fortalecimiento efectivo de los docentes a cargo en el proceso de enseñanza-aprendizaje en el aula con sus alumnos.

En atención a lo anterior, es claro que a pesar de haber avances en torno a las capacidades tecnológicas de las IE oficiales en la región Caribe resulta aún difícil profundizar sobre la inclusión de las TIC en el currículo, por parte de los docentes a nivel de esta región (Lara, Arellano y Said, 2012). Esto aunque se 
hayan hecho fuertes inversiones y apuestas dentro de las políticas, los programas y los proyectos orientados para tal fin.

En el caso de los docentes que enseñan inglés como segunda lengua, autores como Riascos, Ávila y Quintero (2009) destacan la necesidad que éstos tengan conjunto de saberes, conocimientos, destrezas y características individuales que les permita realizar acciones en un contexto determinado. En otras palabras, tener competencias para el ejercicio de esta labor con sus estudiantes. Teniendo en cuenta lo expuesto por los autores antes mencionados y el Ministerio de Educación Nacional de Colombia (2008), algunas de las competencias requeridas por estos docentes son: la competencia comunicativa, la competencia lingüística, la competencia pragmática y la competencia sociolingüística.

El país transita en la mejora de la calidad de la educación, específicamente, en lo que se refiere al área en el que se centra este proyecto: el inglés. De acuerdo con los estándares dispuestos por el MEN (2009), basado en el Marco Común Europeo, la mayoría de los docentes a nivel educativo $72 \%$ alcanza apenas el grado A2 (nivel básico elemental en el conocimiento del inglés), cuando se exige en la actualidad un nivel B2 (nivel superior-intermedio alto para un docente en inglés) (Bastidas y Muñoz, 2011).

\section{Justificación}

Las tecnologías son un recurso fundamental para la práctica pedagógica, sin embargo, su implementación en el entorno educativo es un proceso que implica aceptación, aprendizaje y adaptación, diversos estudios en el mundo evidencia tal premisa.

Según el texto "Estrategias para el Fortalecimiento de las TIC en las Instituciones Educativas Oficiales de Colombia", realizado por el Observatorio de Educación del Caribe Colombiano (OECC) de la Universidad del Norte, se requiere profundizar en el proceso de capacitación y formación de los docentes y directivos con relación a las TIC, teniendo en cuenta el nivel de competencias real con que ellos cuentan. Lo anterior, con la finalidad de estructurar programas acordes y dirigidos al fortalecimiento de competencias no ajustadas en el uso 
operativo de las herramientas tecnológicas, sino a la apropiación de estas de manera significativa (transformadora) a lo largo de todo el proceso de enseñanzaaprendizaje y de gestión ejecutado desde las instituciones educativas. La finalidad, es la de generar la presencia de un mayor número de docentes y directivos que se apropien y usen la tecnología como una herramienta que les potenciaría el desarrollo de sus actividades pedagógicas y supondría una imposición más en cuanto a la forma de enseñar o dirigir un salón de clases o una institución educativa (Valencia et al, 2013).

En consonancia con lo antes planteado, se promueve un mayor y mejor uso de las TIC en los docentes encargados de las labores de enseñanza del inglés desde las instituciones de educación a nivel de básica primaria de la región Caribe de Colombia. Todo esto para la profundización y el conocimiento en los contenidos y contexto de aplicación de las prácticas pedagógicas a cargo de éstos.

Por otra parte, se pretende con el estudio, el estimar la percepción que tienen los docentes a cargo de la enseñanza del inglés a nivel de Básica Primaria en las IE oficiales de la región Caribe de Colombia en cuanto a la utilidad e implementación de las TIC para el ejercicio de sus funciones pedagógicas. Ello con el fin de establecer un mapa de percepciones y de uso relacionado con los recursos TIC actualmente con capacidad de aprovechamiento desde internet, que ayuden a la creatividad y la innovación docente, y hacia al fortalecimiento de la práctica pedagógica en el área de la enseñanza del inglés.

\section{Metodología}

\subsection{Tipo de investigación}

El proyecto está enmarcado dentro del paradigma empírico-positivista y es de carácter descriptivo, debido a que no solo se busca establecer los rasgos que caracterizan la percepción que tienen los docentes en torno al tema aquí planteado, sino los factores que estén incidiendo en éstos, dentro como fuera del aula. Además buscar la identificación de un conjunto de elementos que incide en dicha percepción. 


\subsection{Variables}

- Competencias del uso de las TIC en las docentes de inglés.

- Percepción del uso e importancia de las TIC en las competencias de los docentes de inglés.

\section{3. Área de Estudio}

La escogencia del área geográfica en la que se enmarca este proyecto se basa en torno a los criterios expuestos en el marco del proyecto tomado como guía metodológica para el desarrollo del objetivo general aquí planteado, es decir, el Departamento Atlántico. El estudio se realizó porque:

- Representa el $21 \%$ de la población en edad escolar

- Concentran el $24 \%$ de los establecimientos educativos.

- Concentra el $20 \%$ de los estudiantes matriculados en la Región Caribe.

- Los indicadores de infraestructura TIC y conectividad son más elevados en comparación con los otros departamentos de la Región.

- Representa el $26 \%$ del PIB Regional (a precios constante por departamentos Base 2005).

- Pese a que los datos analizados en este trabajo corresponden solo a docentes del Departamento del Atlántico, la temática y condiciones alrededor de la población de estudio permiten tomar los resultados generales expuestos en este trabajo de fin de maestría como una aproximación de la realidad existente a nivel regional.

En vista que no existen datos fiables en torno al número de docentes encargados en la impartición de la enseñanza del inglés desde las IE oficiales en el Departamento del Atlántico, la población de estudio tomará como base los datos generales que existen desde el MEN para 2010. En lo relacionado con las instituciones educativas oficiales adscritas a las secretarias de educación del Departamento del Atlántico y de las ciudades de Barranquilla, Malambo y Soledad. En total son 313 establecimientos que cumplen con estas características; los cuales atienden a un total de 430.849 estudiantes. 
5.4. Población de estudio y muestra

El proyecto se centró su desarrollo a los docentes a cargo de la enseñanza del inglés en las IE oficiales del Departamento Atlántico. En cuanto al diseño muestral se empleó criterios procedimentales relacionados requeridos para la consecución del objetivo general propuesto.

Para el levantamiento de la información se tomaron los datos del proyecto del que parte esta tesis, a partir de una muestra compuesta por 98 de las 172 instituciones educativas oficiales tomadas en consideración desde el diseño por conglomerado, estratificado en tres etapas (Secretaria, Zona y Sede) con afijación proporcional $^{2}$, aplicada inicialmente. La selección de estas instituciones además de mantener el diseño muestral inicialmente tomado, fue resultado de que en estas instituciones educativas se logró encuestar, durante el proceso de trabajo de campo, a los docentes encargados en la enseñanza del inglés, al encontrarse en la institución y aceptar voluntariamente el diligenciamiento de dicha encuesta. La investigación tomó como guía metodológica, el proyecto financiado por Colciencias en el marco de la convocatoria 578 titulado: 'Medición del impacto de las actividades orientadas al fomento de las TIC en el sector educativo en la Región Caribe Colombiana. Caso Barranquilla y Cartagena ${ }^{3}$. La muestra contó con un nivel de confianza de $95 \%$ y un margen de error (e) de $\pm 5 \%$. La sub-muestra que finalmente fue tomada en nuestro caso contó con un nivel de confianza de $92 \%$ y un margen de error (e) de $\pm \%$ 8; el cual, pese a no ser óptimo, se encuentra en el margen muestral tolerado y aceptado comúnmente en las ciencias sociales. De la población total, que son 172 escuelas oficiales en el Departamento del Atlántico, se tomó una muestra representativa del 57\% equivalente a 98 IED.

\footnotetext{
${ }^{2}$ El diseño muestral que sirvió de referente para la obtención de los datos expuestos en este trabajo de fin de maestría resultó el más adecuado ya que cuando "los sujetos de la población a estudio están organizados en pequeños grupos muy poco homogéneos. Es decir, cuando la población la constituyen agrupaciones naturales como es el caso de una escuela" (Ruiz, 2008, p. 81).

${ }^{3}$ Proyecto dirigido por el Dr. Elias Said Hung, en representación de la Universidad del Norte, como entidad ejecutora principal, en alianza con la Universidad Tecnológica de Bolívar y la Corporación Colombia Digital.
} 
Figura 1. Población de la investigación

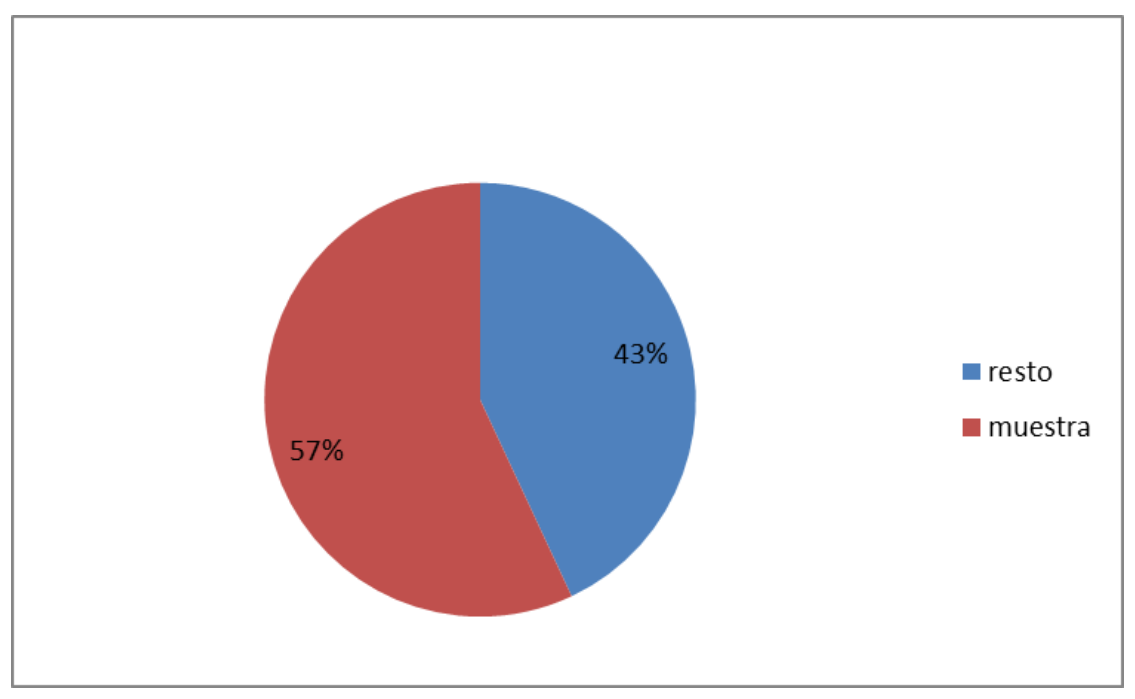

Fuente: Elaboración propia

5.5. Técnicas de investigación aplicadas

Para la consecución del objetivo general y específicos propuestos en este trabajo, se hizo uso de la encuesta, empleada en el marco del proyecto guía de este trabajo de fin de carrera, dirigida a los docentes de IE oficiales del Departamento Atlántico, en vista que cuenta con los marcos de pilotaje que garantizan la validez estadística y de constructo para el abordaje temático aquí planteado, con la validación externa del asesor internacional vinculado al proyecto para el análisis del tema tratado en esta tesis de fin de maestría.

La investigación es tipo descriptiva, en el cual, se hizo un planteamiento del problema, los objetivos, la justificación, teoricidad, el diseño metodológico y al final se establecieron las conclusiones y recomendaciones derivadas de la investigación. Se hizo una recolección de datos sobre los municipios del departamento del Atlántico, con una población de 243 docentes bilingües de la básica primaria en las escuelas públicas de cada uno los municipios. Los instrumentos que se utilizaron durante el método cuantitativo fueron las encuestas impresas y usando finalmente el software estadístico SPSS para la explotación de datos. 


\section{Análisis de Resultados}

A continuación se muestran los resultados obtenidos en el trabajo de campo acorde con cada uno de los objetivos planteados en la investigación.

- Identificar el perfil de uso de las TIC de los docentes a cargo de la enseñanza del inglés a nivel de Básica Primaria en las IE oficiales de la región Caribe de Colombia.

Figura 2. Perfil del uso de las TIC de los docentes de inglés a nivel básica primaria
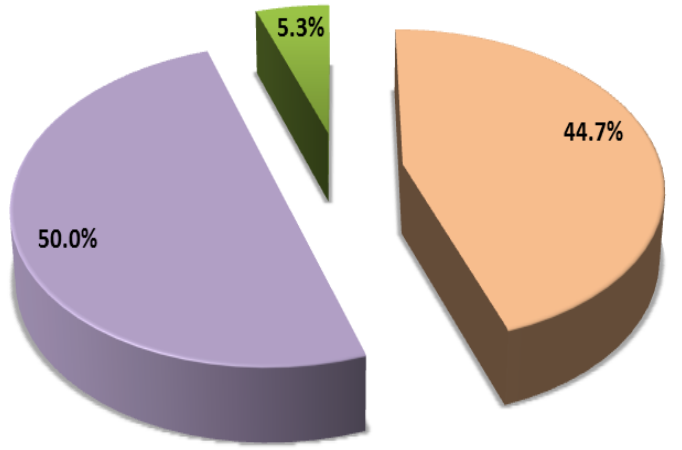

$\square \mathrm{SI}$

$\square \mathrm{NO}$

$\square \mathrm{NS} / \mathrm{NC}$

Fuente: Elaboración propia

El $44,7 \%$ de los docentes encuestados, no ha asistido a algún curso de formación en el uso de TIC en el último año, el 50\% si lo ha hecho y el 5,3\% no sabe o no conoce.

Figura 3. Cursos de formación realizados por los docentes

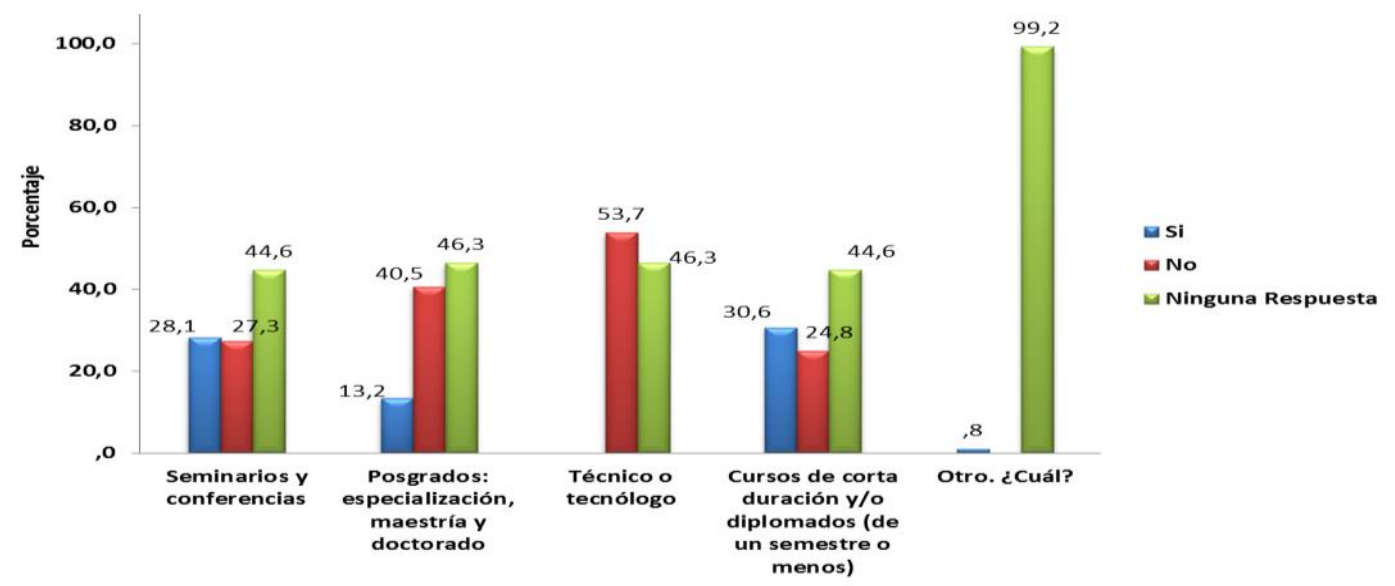

Fuente: Elaboración propia 
Los docentes que han asistido a algún curso de formación en el uso de TIC en el último año. El 30.6\% han sido cursos de corta duración y/o diplomados (de un semestre o menos), seguido del $28,1 \%$ que han asistido a seminarios y conferencias. Un 13,2\% han realizado algún Postgrado (Especialización, Maestría y Doctorado).

- Establecer la percepción que tienen los docentes a cargo de la enseñanza del inglés a nivel de Básica Primaria en las IE oficiales de la región Caribe de Colombia, en torno a la utilidad e implementación de las TIC en la labor de profundización y generación de conocimiento en los contenidos y contexto requeridos para el desarrollo de sus prácticas pedagógicas.

Dentro de las actividades o acciones calificadas dentro del rango de ALTO por ellos mismo se pueden destacar las siguientes:

- $\quad$ Buscar y seleccionar información utilizando diferentes herramientas TIC y fuentes como buscadores, bibliotecas virtuales, repositorios, etc. con un $61,78 \%$.

- Establecer comunicación con otros utilizando TIC a través de email, chat foros, mensajes de texto, etc. Con un 60,9\%. Como se aprecia en la siguiente figura:

Figura 4. Percepción que tienen los docentes sobre las TIC en la enseñanza del inglés a nivel de Básica

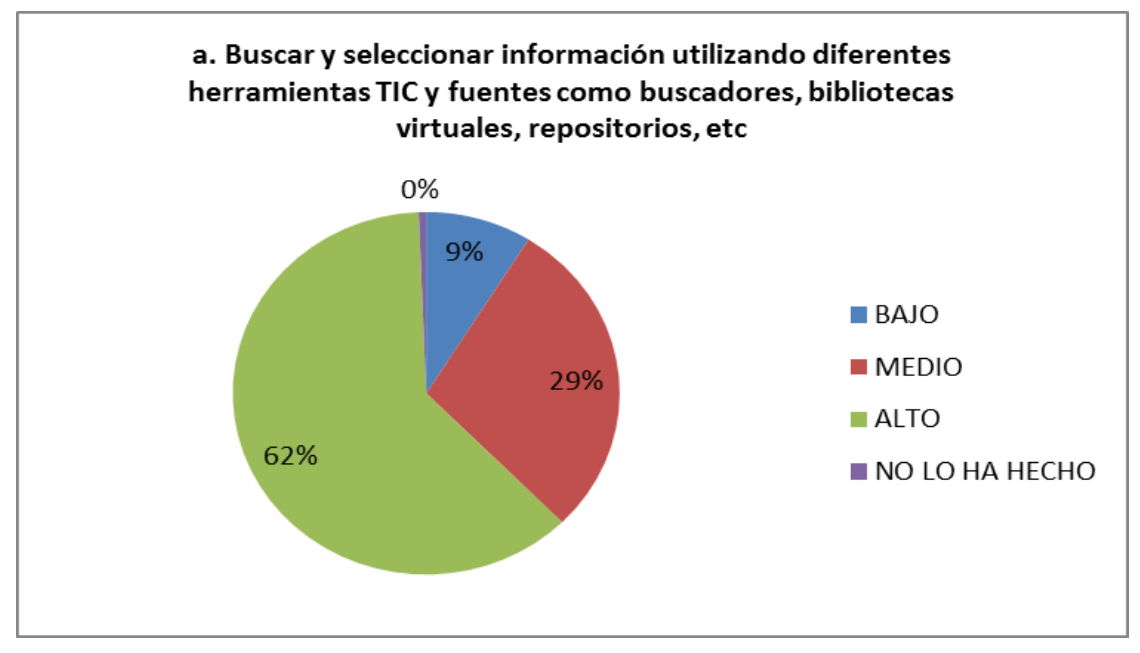

Fuente: Elaboración propia 
Figura 5. Comunicación con otros utilizando las TIC

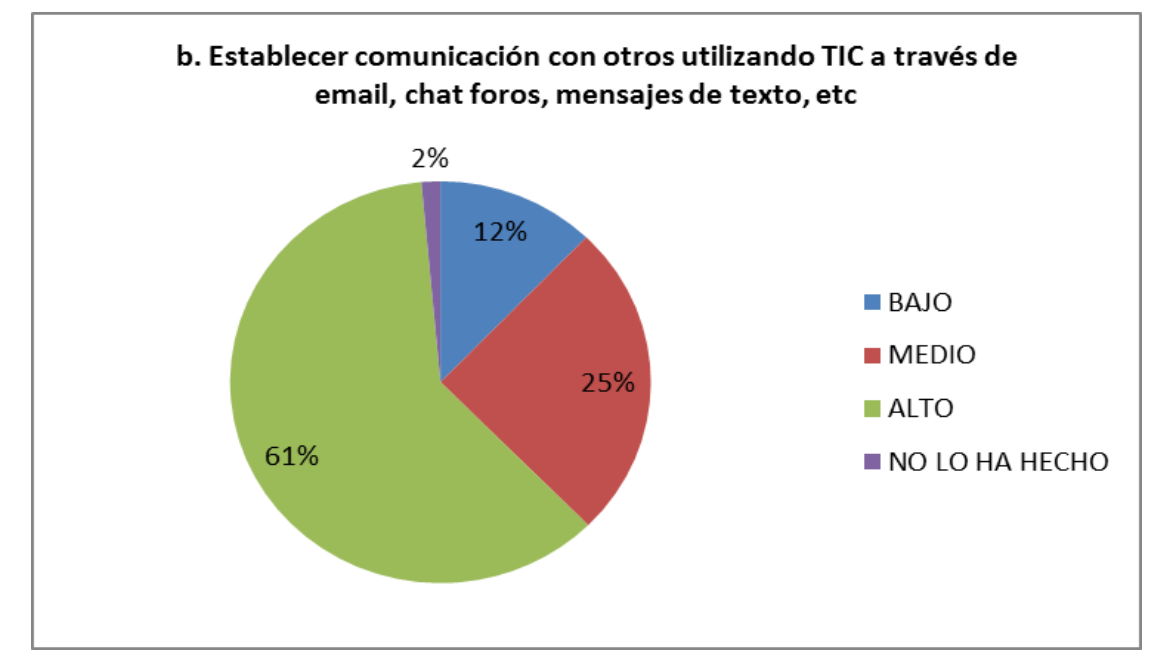

Fuente: Elaboración propia

- Determinar los rasgos que caracterizan el uso de las TIC en las prácticas pedagógicas, ejercidas por los profesores estudiados.

En la evaluación del desempeño, el crecimiento profesional y el liderazgo de los docentes, se proponen varias opciones para que cada uno de ellos, identifique cuales se ajustan mejor a su perfil. Los resultados evidencian que el $65.7 \%$ aproximadamente indaga y reflexiona sobre el uso de las TIC a favor de las necesidades de aprendizaje de los estudiantes. De igual manera, existen opciones que con altas representatividades como se evidencia en la siguiente gráfica: 
Figura 6. Los rasgos que caracterizan el uso de las TIC en las prácticas pedagógicas

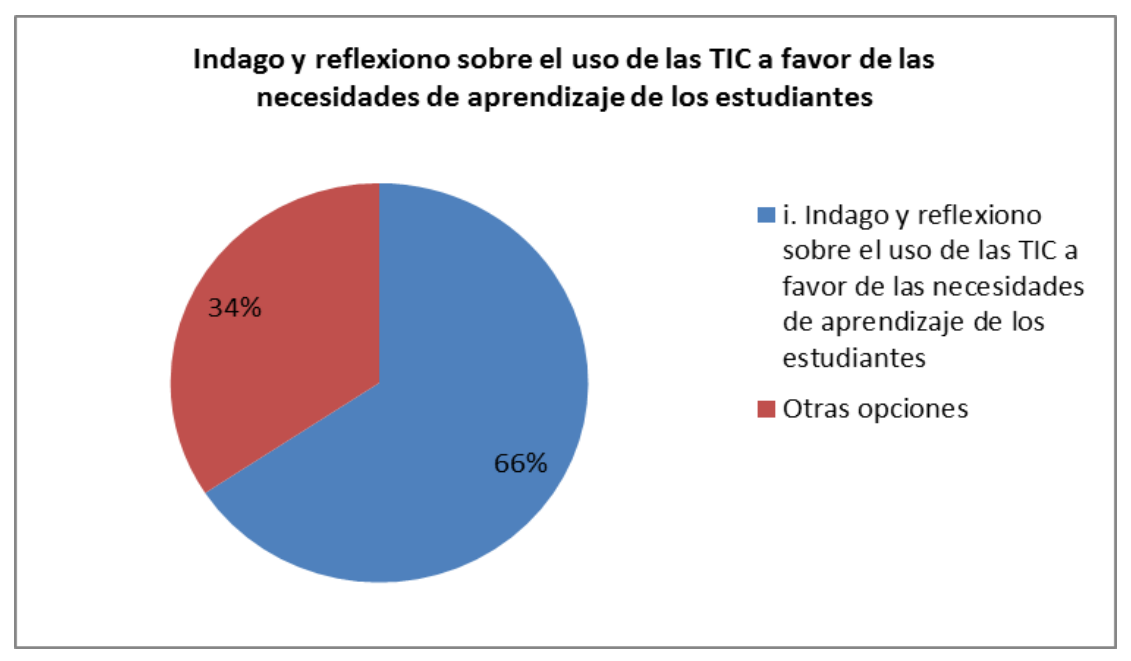

Fuente: Elaboración propia

Lo anterior revela que el escenario principal del docente para la enseñanza de los contenidos pedagógicos, apoyados con las nuevas tecnologías, son el área de informática, limitando el horario y la flexibilidad del currículo escolar.

- Identificar los niveles de percepción que tienen los docentes en torno a los niveles de competencias vinculadas con la profundización y generación de conocimiento de contenidos desde las TIC.

El resto de opciones con las que se identifican los docentes, se relacionan a continuación con sus respectivas participaciones porcentuales:

- El 80\% de los docentes encuestados para estas instituciones, están de acuerdo con que las TIC han ayudado a transformar las formas de comunicación y las relaciones sociales.

- El 85,1\% considera que las TIC aportan mejoras a la sociedad.

- El 83,5\% de ellos considera que El uso de las TIC es indispensable sus clases.

- El 88,4\% consideran que es importante actualizarse en el uso educativo de TIC, entre otras. 
En síntesis, se puede observar que una gran mayoría de los docentes encuestados están de acuerdo con el uso, actualización, capacitación y manejo de las TIC tanto en su vida cotidiana como en el desarrollo de sus actividades pedagógicas en el ambiente educativo.

\section{Conclusiones}

De la presente investigación, se establecieron las siguientes conclusiones:

- Los docentes son conscientes de que las TIC son recursos clave dentro del proceso educativo actual, y pueden apoyar la enseñanza y el aprendizaje de segunda lenguas; sin embargo, el desconocimiento sobre las potencialidades de estas herramientas hace que los docentes se les dificulta su aplicación didáctica en el aula de clase.

- Surge la necesidad de que haya un mayor apoyo por parte del Estado y las instituciones educativas en cuanto a lograr una integración significativa y efectiva de las tecnologías en las situaciones educativas.

- La profundización del conocimiento, está referida a que el docente posea las competencias necesarias para integrar metodologías didácticas y TIC más sofisticadas, enfatizando en la comprensión del conocimiento escolar y sobre todo su aplicación tanto a problemas del mundo real como a su propio abordaje pedagógico.

- La generación de conocimiento, implica el desarrollo de la innovación, en el cual, mediante la aplicación de las TIC, se busca producir un nuevo conocimiento y sacar provecho de éste. Así como fomentar la participación cívica, la creatividad cultural y la productividad en función no solo del aprendizaje de un saber específico, sino también, conocimiento para generar cambios en los modelos de enseñanza-aprendizaje tradicionales. Se busca facilitar los procesos de enseñanza de los estudiantes de los modelos pedagógicos.

- La inclusión de las TIC en numerosos centros educativos de la región Caribe debe estar articulada con los aspectos misionales, objetivos pedagógicos y las necesidades de los estudiantes. Esto implica que la 
formación del docente, este referida a desarrollar las destrezas y habilidades en el manejo de los recursos tecnológicos. Además, potencializar las ventajas educativas que éstos recursos ofrecen en cuanto a los procesos de enseñanza y aprendizaje, por ello, su aplicación supone un factor fundamental de cambio formativo tanto dentro como fuera del aula de clase.

- En lo referente a la enseñanza del inglés, mediante la aplicación de las TIC, el trabajo de campo nos revela que los docentes sí valoran el uso de las TIC en el proceso pedagógico de enseñanza del inglés; aunque, la falta de conocimiento sobre el manejo apropiado de estas herramientas tecnológicas, y sobre las ventajas educativas que están brindan, hacen que haya una subutilización en la aplicación de las TIC en el proceso educativo en estas escuelas de primaria en la región Caribe colombiana.

- El trabajo de campo nos muestra que a nivel del Departamento del Atlántico, los docentes no están generando un nuevo conocimiento, debido a que no participan en la construcción de proyectos investigación apoyados por tecnologías. Los docentes en su mayoría utilizan las herramientas TIC para buscar información para sus contenidos pedagógicos y no en el sentido de fortalecer los procesos de investigación para el desarrollo de proyectos de conocimiento.

- De los hallazgos obtenidos en la investigación, se desprende que los bajos niveles de formación profesional que presentan los docentes para su enseñanza pedagógica; obedecen a razones de deserción, desinterés y desconocimiento por parte de los mimos. De igual forma, desconocen las políticas públicas en TIC que apoyen el uso de las tecnológicas en las competencias pedagógicas. También estos bajos niveles de preparación, están en concomitancia con el difícil acceso que tienen los docentes a la aplicación de estos estudios de posgrado, ya que los estándares en exámenes reglamentados por el Ministerio de Educación Nacional y los requisitos de las becas ofrecidas por el Estado colombiano tienen un nivel de conocimiento muy alto, es decir, que tales pruebas son realizadas con 
base en estándares internacionales, en las que los niveles de formación son superiores.

- Otro aspecto singular en la investigación, es como los docentes carecen de conocimiento sobre el uso de las TIC en los dispositivos móviles, por lo cual, sele dificultad para trabajar sus contenidos pedagógicos. Por otra parte, algunas bibliotecas no cuentan con una infraestructura adecuada para que los docentes puedan buscar los contenidos pedagógicos digitales y hacer un uso apropiado de las tecnologías.

- En relación con los centros de Internet públicos, son relativamente costosos y no están dotados con la infraestructura necesaria para el desarrollo de su trabajo. Por lo tanto, la opción de trabajar desde su casa se vuelve más aceptable, en el sentido, de que el docente adapta sus equipos a las necesidades de información y de conocimiento, y puede trabajar de manera autónoma desde su hogar.

- En relación a facilitar la enseñanza del inglés, las TIC permiten la creación de entornos virtuales que fortalecen la clase magistral. Los estudiantes puedan interactuar en línea con otros, desarrollar trabajo colaborativo y tener un acompañamiento personalizado a través de web, el desarrollo de la innovación, creatividad, y generación de conocimientos en TIC en el diseño de entornos virtuales.

- Las herramientas tecnológicas en el aprendizaje del área de inglés permiten profundizar en aquellos componentes audiovisuales que incrementan el grado de aprehensión superior frente a la clase presencial. Lo anterior, está referido a que se desarrolle el aprendizaje del idioma del inglés a través de los procesos cognitivo y cognoscitivos, para ello el docente debe estar capacitado y este debe ser un mediador del proceso.

- La aplicación de las TIC es una ventaja educativa que facilita la efectividad del proceso pedagógico, y en cual, se tiene claro que no es el mero uso de las tecnologías lo que produce un efecto beneficioso, sino su integración significativa en las prácticas concretas, atendiendo a las necesidades y particularidades de cada grupo y cada situación. También la investigación 
pone de relieve, como el docente logra identificar la importancia que tienen las TIC dentro del proceso educativo, como medio de expresión, fuente abierta de información, instrumento para procesar la información, canal de comunicación presencial, canal de comunicación virtual, medio didáctico, herramienta para la evaluación, diagnóstico y rehabilitación. Además generar espacios formativos, entre otros aspectos, que facilitan la labor docente, así como también, permiten la realización de nuevas actividades de aprendizaje y suponen el aprendizaje de nuevos conocimientos y competencias (Cabero, 2004).

Otro aspecto a concluir, es como el interés del docente en el uso de las TIC se centra en la búsqueda de información, es decir, como soporte de la investigación. En menor orden de importancia, está el desarrollo de experiencias de aprendizaje personalizado acorde con las necesidades y perfiles del estudiante; que exista una mayor autonomía en el uso de las herramientas de las TIC.

\section{Referencias Bibliográficas}

Bonilla, J. (et al). (2006). La educación frente al TLC. Universidad Santo Tomas de Aquino, Contaduría Pública, Bogotá, D.C. Recuperado de http://administracion.univalle.edu.co/Comunidad/Memorias/evento3/archivos/Educ

\section{acion\%20en\%20el\%20TLC.pdf}

Said, E. (2014). Medición del Impacto de las actividades orientadas al fomento de las TIC en el Sector Educativo en la Región Caribe Colombiana. Universidad del Norte, Departamento de Comunicación Social, División de Humanidades y Ciencias Sociales e Instituto de Estudios en Educación.

Bastidas, J. y Muñoz, G. (2011). Fundamentos para el desarrollo profesional de los profesores de Inglés. Pasto: Graficolor.

Lara, J., Arellano, W., y Said, E. (2012). Las TIC en los currículos de las Instituciones Educativas Oficiales de la Región Caribe Colombiana. Caso Barranquilla $y$ Cartagena. Recuperado en: http://www.virtualeduca.red/documentos/23/ARTICULO\%20CIENTIFICO\%20578\% 20LAS\%20TIC\%20EN\%20LOS\%20CURRÍCULOS\%20DE\%20INSTITUCIONES\% 20EDUCATIVAS\%20OFICIALES.pdf 
Llorente, C. y Cabero, A. (2010). Desarrollo de un Instrumento sobre Competencias TIC en alumnos Universitarios. Alfabetización mediática y culturas digitales. Ponencia presentada en el Congreso Euro-Iberoamericano ATEI 2010. Sevilla: Universidad de Sevilla, España.

Mejía, N. y Puche, L. (2008). Generación de Conocimientos en TIC: Caso UNIGUAJIRA. Recuperado de

http://www.virtualeduca.info/ponencias2013/113/GeneracindeConocimientosenTIC CasoUNIGUAJIRA.docx

Ministerio de Educación Nacional. (2013). Competencias TIC Para el Desarrollo Profesional Docente. Bogotá, D.C: Ministerio de Educación Nacional. Recuperado http://www.colombiaaprende.edu.co/html/micrositios/1752/articles-

\section{4 recurso tic.pdf}

Riascos, S., Ávila, G., y Quintero, M. (2009). Las TIC en el aula: percepciones de los profesores universitarios. Educación y Educadores, 12(3), 133-157. Recuperado de file:///C:/Users/Adriano/Downloads/1536-6375-1-PB.pdf

Robinson, K. (2011). Out of Our Minds: Learning to be creative. Oxford, Reino Unido: Capstone.

Sigalés, C., Mominó, J., Meneses, J., y Badía, A. (2008). La integración de internet en la educación escolar española: situación actual y perspectivas de futuro. Barcelona: UOC. Recuperado de

http://www.uoc.edu/in3/integracion internet educacion escolar/esp/pdf/informe e scuelas.pdf

UNESCO. (2008). Estándares de competencia en TIC para docentes. Londres: UNESCO. Recuperado de http://www.oei.es/tic/UNESCOEstandaresDocentes.pdf

UNESCO. (2011). UNESCO ICT Competency Framework for Teachers. Paris: UNESCO and Microsoft. Recuperado de http://unesdoc.unesco.org/images/0021/002134/213475e.pdf 
GiovanNi PolifRoni LoBo

Valencia, J. y Camargo, K. (2013). Estrategias para el fortalecimiento de las TIC en las Instituciones Educativas Oficiales de Colombia. Bogotá, D.C., Colombia:

Colombia Digital. Recuperado de

file:///C:/Users/Adriano/Downloads/estrategias fortalecimiento tic.pdf 